\title{
Migratory pulmonary infiltrates
}

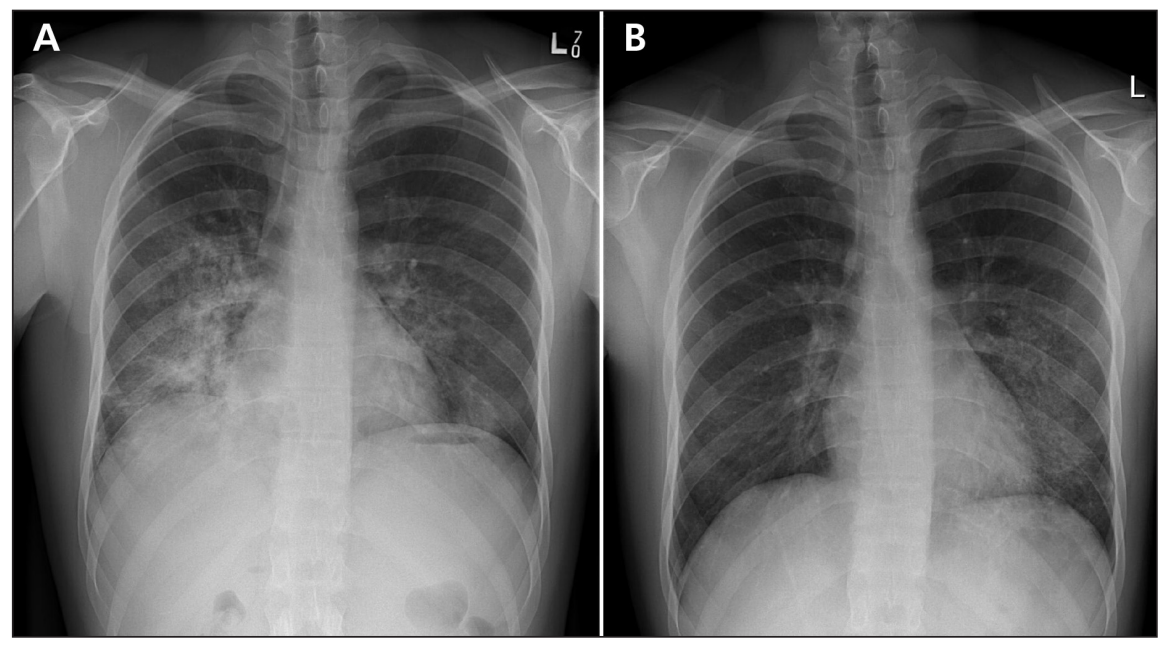

Figure 1: Chest radiographs taken (A) 2 weeks before evaluation showing pulmonary infiltrates in the lower regions of both lungs, and (B) during evaluation showing improvement in pulmonary infiltrates on the right side and worsening of infiltrates on the left side.

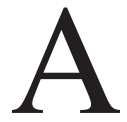

24-year-old man presented with severe anemia (hemoglobin $62 \mathrm{~g} / \mathrm{L})$. He had a 1 month history of dry cough, chest pain that was intermittent and pleuritic, and progressive breathlessness on exertion. The patient reported having occasional chills and night sweats. He denied weight loss or hemoptysis. A radiograph of his chest taken 2 weeks before presentation had shown pulmonary infiltrates in the lower regions of both lungs (Figure 1A). He had subsequently completed a 10-day course of moxifloxacin. The patient was a smoker. He also reported that, 2 to 3 years before, he had engaged in many episodes of unprotected sex with a male partner who was discovered to have multiple other sexual partners during the same period.

On examination, the patient was pale and had tachycardia. We found no cyanosis or clubbing. His oxygen saturation was $96 \%$ by pulse oximetry while breathing ambient air. His lungs were clear to auscultation. Laboratory tests showed a hemoglobin concentration of $62 \mathrm{~g} / \mathrm{L}$ (mean corpuscular volume $82 \mathrm{fL}$ ) and a reticulocyte count of $84 \times 10^{9} / \mathrm{L}$. The ferritin level $(86 \mu \mathrm{g} / \mathrm{L})$ and the serum creatinine level (108 $\mu \mathrm{mol} / \mathrm{L}$ ) were within normal limits. His leukocyte count differential was also normal. His lactate dehydrogenase level, total bilirubin level, international normalized ratio and partial thromboplastin time were within normal limits. A chest radiograph showed a decrease in the pulmonary infiltrates on the right side and an increase on the left side (Figure 1B).

\section{What is the next most appropri- ate diagnostic test or procedure?}

a. Computed tomography scan of the chest

b. Urinalysis

c. HIV test

d. Bronchoscopy with bronchoalveolar lavage

e. Further testing for hemolysis, including testing for cold agglutinins

All of these investigations might be considered appropriate at this point. In our patient's case, the tests that led to the actual diagnosis were (b) and (d).

After being given a blood transfusion, the patient felt well and was discharged. We arranged for close followup on an outpatient basis to assess his symptoms, HIV status and hemoglobin

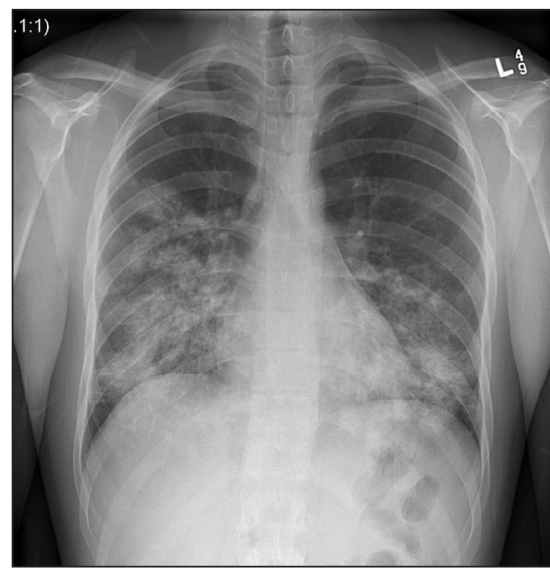

Figure 2: Chest radiograph taken 6 weeks after initial evaluation showing new pulmonary infiltrates in the right mid-lung zone and worsening infiltrates in the lower lung zones on both sides.

level. We administered a course of azithromycin for a presumptive diagnosis of mycoplasma pneumonia, possibly associated with hemolytic anemia due to cold agglutinins or anemia secondary to HIV infection. The HIV test result was reported 1 week later and was negative.

The patient presented again 6 weeks later with the same symptoms. This time his hemoglobin level was $73 \mathrm{~g} / \mathrm{L}$, his serum creatinine was $118 \mu \mathrm{mol} / \mathrm{L}$, and a urinalysis showed proteinuria $(3+)$ and microscopic hematuria (5+). No casts were present. A chest radiograph showed worsening of the pulmonary infiltrates on both sides (Figure 2).

\section{What is your diagnosis?}

a. Pneumocystis jiroveci infection

b. Löffler syndrome

c. Goodpasture syndrome

d. Churg-Strauss syndrome

e. Cryptogenic organizing pneumonia 


\section{WHAT IS YOUR CALL?}

\section{Discussion}

The diagnosis is (c) Goodpasture syndrome. The presence of risk factors for HIV infection led us to focus initially on infectious causes. After HIV infection was ruled out, we discovered significant microscopic hematuria which raised the possibility of a pulmonaryrenal syndrome. Bronchoscopy with bronchoalveolar lavage showed diffuse alveolar hemorrhage. Tests for antinuclear antibodies and antineutrophil cytoplasmic autoantibodies were negative. However, the titre of antiglomerular basement membrane antibodies was elevated at 1:40. Renal biopsy showed lesions that were segmental and necrotizing with cellular crescents (Figure 3A). Linear staining of glomerular basement membranes was strongly positive for IgG (Figure 3B). Both the lesions and the linear staining features are diagnostic of Goodpasture syndrome.

The patient was administered prednisone and cyclophosphamide and underwent a series of 9 plasma-exchange treatments. Test results for antiglomerular basement membrane antibodies were negative after 3 months of therapy. After 6 months of follow-up, the patient had no symptoms and his serum creatinine level had decreased to within normal limits.

Goodpasture syndrome is rare, affecting fewer than 1 person per million. ${ }^{1}$ Autoantibodies directed against the glomerular basement membrane are produced in response to an unknown stimulus and cause glomerulonephritis. In about $60 \%$ of cases, they also cause pulmonary hemorrhage by targeting antigens in the alveolar basement membrane. Cigarette smoking increases the risk of pulmonary involvement. ${ }^{2}$ The lungs are affected more frequently in younger adults.

Patients with the pulmonary manifestations of Goodpasture syndrome present with dyspnea and cough. ${ }^{3}$ Hemoptysis occurs less frequently. Pulmonary infiltrates are frequently migratory, and iron deficiency anemia may occur.

The term "migratory" is used to describe recurrent pulmonary infiltrates that appear and resolve over a short pe-

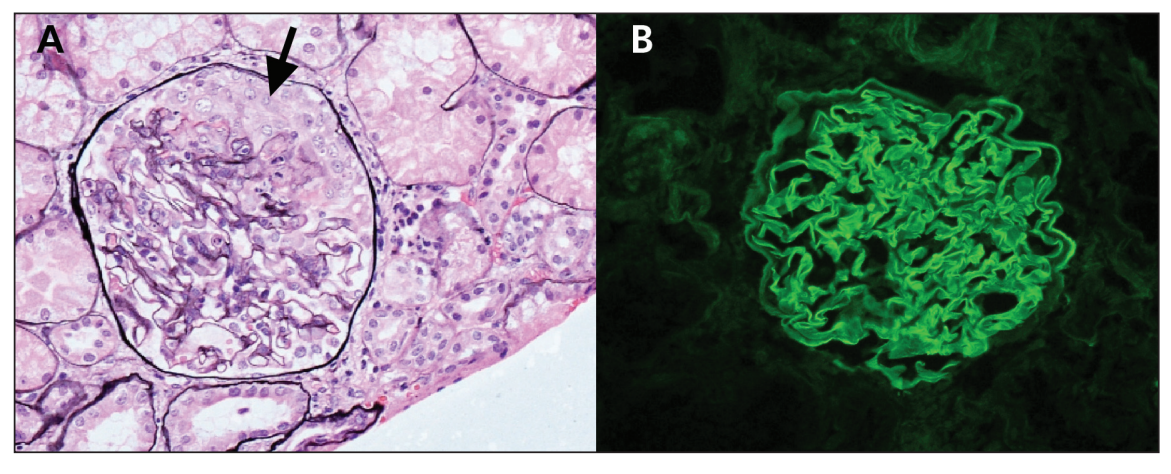

Figure 3: (A) A renal biopsy specimen stained with silver methenamine showing proliferating epithelial cells in a crescent form within the glomerulus (arrow), the characteristic morphology of rapidly progressive glomerulonephritis. (B) Immunofluorescent stain for IgG showing linear staining of the glomerular basement membrane, a sign of antiglomerular basement membrane antibodies.

riod (days to weeks), with new infiltrates developing elsewhere in the lungs. The infiltrates thus appear to move or "migrate" through the lungs over time.

Pulmonary function testing in patients with Goodpasture syndrome shows an elevated diffusing capacity for carbon monoxide. The lung is more likely to be affected if there is antecedent parenchymal injury caused by factors such as infection or, more frequently, cigarette smoking. Constitutional symptoms of fatigue, weight loss and fever are rare and often suggest an alternate cause such as vasculitis.

A diagnosis of Goodpasture syndrome is based on the findings of tests for antiglomerular basement membrane antibodies and from a renal biopsy. The biopsy will show linear IgG deposits along the glomerular basement membrane, which are pathognomonic for the disorder (Figure 3B). Treatment consists of high-dose prednisone and cyclophosphamide therapy in combination with plasmapheresis. However, limited evidence is available to support this treatment. ${ }^{4}$ Long-term outcomes for Goodpasture syndrome depend on the initial degree of renal impairment and the severity of histologic findings.

Three learning points arise from this case. First, pulmonary hemorrhage is an important cause of severe anemia and should be considered in the differential diagnosis. An absence of hemoptysis does not rule out significant pulmonary hemorrhage.

Second, relatively small rises in serum creatinine levels can imply a significant loss of renal function. Our patient's serum creatinine level was elevated only minimally; his pulmonary symptoms were much more prominent. Therefore, we did not initially consider the possibility of a pulmonary-renal syndrome. Our example highlights the importance of recognizing the relatively poor sensitivity of serum creatinine levels for detecting early reductions in glomerular filtration rate in acute kidney injury. Serum creatinine levels change little when the glomerular filtration rate is reduced from $120 \mathrm{~mL} / \mathrm{min}$ to $90 \mathrm{~mL} / \mathrm{min}$. But it rises significantly with a reduction in the glomerular filtration rate from $90 \mathrm{~mL} / \mathrm{min}$ to $60 \mathrm{~mL} / \mathrm{min}$. Therefore, clinicians should consider directly assessing the glomerular filtration rate by measuring creatinine clearance when any uncertainty exists about the significance of the serum creatinine value.

Third, urinalysis should be included in any assessment because it is fast, inexpensive and often informative. We would have been more likely to consider a pulmonary-renal syndrome in our patient's case had we been aware of the microscopic hematuria.

In short, pulmonary hemorrhage should be considered in any patient presenting with recurrent pulmonary infiltrates, particularly if there is unexplained anemia.

\section{Diagnostic testing}

Considerations when selecting a diagnostic test include not only the relevance of the result to the clinician's ability to rule in or out important diagnostic hy- 
potheses (i.e., probable or life-threatening conditions) and the expense of the test, but also the ease and speed with which the test may be performed. An HIV test was certainly indicated in this case, but the results would not have been immediately available for use. A peripheral blood film would have been helpful in narrowing the differential diagnosis if the patient's anemia was thought to be related to hemolysis. However, the markers of hemolysis were otherwise reassuring. Urinalysis is an inexpensive, easy and rapid test that allows a clinician to assess the possibility of a pulmonaryrenal syndrome when evaluating a patient with unexplained pulmonary infiltrates. Therefore, it is the next best test.
Computed tomography scanning and bronchoscopy are more expensive procedures that should be considered at some point to aid in diagnosis but will not narrow the differential diagnosis as efficiently.

Differential diagnosis: Pneumocystis jiroveci infection is a common opportunistic pulmonary infection in patients with poorly controlled HIV infection (Table 1). It typically presents with an insidious onset of dyspnea, dry cough and constitutional symptoms associated with bilateral pulmonary infiltrates. In our patient's case, the absence of risk factors for immunocompromise (e.g., long-term corticosteroid therapy or use of other im-

Table 1: Differential diagnosis of Goodpasture syndrome

\begin{tabular}{|c|c|}
\hline Condition & Characteristics \\
\hline $\begin{array}{l}\text { Pneumocystis } \\
\text { jiroveci } \\
\text { infection }\end{array}$ & $\begin{array}{l}\text { Occurs in immunocompromised patients. Patients may present } \\
\text { with subacute onset of cough, dyspnea, hypoxemia and bilateral } \\
\text { pulmonary infiltrates in a "bat wing" distribution. Often } \\
\text { associated with an elevated serum lactate dehydrogenase level. } \\
\text { Diagnosis is based on microscopic examination of samples of } \\
\text { bronchoalveolar lavage fluid. }\end{array}$ \\
\hline $\begin{array}{l}\text { Goodpasture } \\
\text { syndrome }\end{array}$ & $\begin{array}{l}\text { Pulmonary-renal syndrome characterized by rapidly progressive } \\
\text { glomerulonephritis and diffuse alveolar hemorrhage. Pulmonary } \\
\text { symptoms accompanied by acute renal failure or hematuria } \\
\text { (gross or microscopic) should raise clinical suspicion. Patients are } \\
\text { typically younger adults and frequently report a history of } \\
\text { cigarette smoking. }\end{array}$ \\
\hline $\begin{array}{l}\text { Löffler } \\
\text { syndrome }\end{array}$ & $\begin{array}{l}\text { The transpulmonary passage of helminth larvae produces a } \\
\text { prototypical syndrome of transient pulmonary infiltrates and } \\
\text { peripheral blood eosinophilia. Ascaris lumbricoides infection is } \\
\text { the most common cause worldwide; hookworms and } \\
\text { Strongyloides stercoralis may also produce the syndrome. } \\
\text { Patients frequently complain of an irritating, nonproductive } \\
\text { cough and burning substernal discomfort aggravated by } \\
\text { coughing or deep breathing. Sometimes dyspnea, wheezing and } \\
\text { blood-tinged sputum occur. }\end{array}$ \\
\hline $\begin{array}{l}\text { Churg-Strauss } \\
\text { syndrome }\end{array}$ & $\begin{array}{l}\text { Small-vessel vasculitis mediated by antineutrophil cytoplasmic } \\
\text { autoantibodies that is associated with peripheral eosinophilia. It } \\
\text { causes a clinical syndrome affecting the skin, joints, heart and } \\
\text { peripheral nerves. It is also a well-described cause of pulmonary- } \\
\text { renal syndrome. Patients with pulmonary disease classically } \\
\text { present with severe, refractory asthma-like symptoms. } \\
\text { Pulmonary hemorrhage is unusual. }\end{array}$ \\
\hline $\begin{array}{l}\text { Cryptogenic } \\
\text { organizing } \\
\text { pneumonia }\end{array}$ & $\begin{array}{l}\text { Idiopathic inflammatory process of the small airways and alveoli. } \\
\text { By definition, it is the pathologic equivalent of bronchiolitis } \\
\text { obliterans with organizing pneumonia (BOOP), which occurs in } \\
\text { the setting of underlying infectious or immune disorders. } \\
\text { Clinical presentation frequently mimics that of community- } \\
\text { acquired pneumonia. Pulmonary infiltrates are frequently } \\
\text { migratory and usually occur bilaterally in peripheral lung zones. } \\
\text { Diagnosis relies on characteristic histopathologic changes in a } \\
\text { lung biopsy sample in the absence of any known secondary } \\
\text { causes. }\end{array}$ \\
\hline
\end{tabular}

munosuppressive medications) and the negative HIV test result ruled out the possibility of $P$. jiroveci infection.

Löffler syndrome consists of transient migratory pulmonary infiltrates that are associated with peripheral eosinophilia. It originates in the transpulmonary passage of helminth larvae, most notably ascariasis. Our patient was an unlikely candidate for this diagnosis given the absence of peripheral eosinophilia and a lack of risk factors for parasitic infection.

Patients with the pulmonary manifestations of Churg-Strauss syndrome classically present with severe, refractory asthma-like symptoms. The absence of typical clinical features or peripheral eosinophilia, together with the negative test result for antineutrophil cytoplasmic autoantibodies, ruled out this diagnosis.

Cryptogenic organizing pneumonia is an inflammatory disorder of the small airways, alveolar ducts and alveoli that frequently mimics communityacquired pneumonia in its presentation. It is a diagnosis of exclusion. The pulmonary hemorrhage that we found on bronchoalveolar lavage confirmed an alternate diagnosis of diffuse alveolar hemorrhage.

\section{Ewan C. Goligher MD \\ Department of Medicine \\ Allan S. Detsky MD PhD \\ Department of Medicine and \\ Department of Health Policy \\ Management and Evaluation \\ University of Toronto \\ Toronto, Ont.}

This article has been peer reviewed.

Competing interests: None declared.

\section{REFERENCES}

1. Bolton WK. Goodpasture's syndrome. Kidney Int 1996;50:1753-66.

2. Donaghy M, Rees AJ. Cigarette smoking and lung hemorrhage in glomerulonephritis caused by autoantibodies to glomerular basement membrane. Lancet 1983;2:1390-2.

3. Ball JA, Young KR Jr. Pulmonary manifestations of Goodpasture's syndrome, antiglomerular basement membrane disease and related disorders. Clin Chest Med 1998;19:777-91.

4. Levy JB, Turner AN, Rees AJ, et al. Long-term outcome of anti-glomerular basement membrane antibody disease treated with plasma exchange and immunosuppression. Ann Intern Med 2001;134: 1033-42. 